\title{
SCIENTIFIC REPORTS

\section{Determination of Kaurenoic Acid in Acanthopanax trifoliatus by Ultra-High Performance Liquid Chromatography Coupled with Tandem Mass Spectrometry (UHPLC-MS/MS)}

\author{
Qun Peng ${ }^{1,3}$, Jianyuan Chen ${ }^{2,3}$, Hanying Duan ${ }^{1 *} \&$ Chao Wang ${ }^{1 *}$
}

Acanthopanax trifoliatus (L.) Merr. (A. trifoliatus) belongs to the family Araliaceae, which is called "Le Cai", and is an indigenous plant to Guangdong Province that has been prevalently planted for years. $A$. trifoliatus is used in folk medicine and has ginseng-like activity. Kaurenoic acid ((-)-kaur-16-en-19-oic acid, KA) is a kaurane-type diterpenoid that is regarded as a major compound in A. trifoliatus. Early studies have reported the determination of KA by HPLC capillary electrophoresis. However, KA could not be completely separated from other components in the plant extract by HPLC because of their similar molecular structures and physical and chemical properties. UHPLC-MS/MS could be a useful tool to identify and quantify KA. In the present work, a UHPLC-ESI-MS/MS method for determining KA in $A$. trifoliatus was developed and validated. KA was extracted from lyophilized $A$. trifoliatus leaves by ultrasound-assisted extraction and further purified by solid phase extraction (SPE). KA was quantified and separated on an Accucore $\mathrm{C}_{18}$ LC column. Mass spectrometry with multi-reaction monitoring (MRM) and quantitative fragment ion/product ion (m/z: 301.3/301.3) in ESI negative mode was used for quantification. The intra-assay and inter-assay relative standard deviation (R.S.D.) were $2.8 \%$ and 3.2\%, respectively. The inter-person R.S.D. on the same day was 3.6\%. The inter-instrument R.S.D. with the same model on the same day was $2.9 \%$. The recoveries evaluated upon spiking three different concentrations of KA were above $97 \%$. A minor matrix effect of $94 \%$ was observed. This method has been applied successfully for the determination of KA in $A$. trifoliatus leaves.

Acanthopanax is a plant genus that embraces 18 plant species that are mainly distributed in Asia and the far-eastern region of Russia ${ }^{1,2}$. One of the Acanthopanax species is A. trifoliatus, which is called "Le Cai" in Chinese, and is an indigenous plant in Enping, Guangdong Province that has been prevalently planted for years. The planting area has reached 420 hectares, and the total value is worth approximately 120 million. A. trifoliatus, with ginseng-like activity, has been used in folk medicine in southern China since old time to treat sinew, bone pains, rheumatism, bruises, neuralgia, impotence, gout, hepatitis and diabetes ${ }^{3-5}$. In Cambodia, Laos, and Vietnam, A. trifoliatus has been used to treat nervous affections and improve memory ${ }^{6,7}$. It has also been shown to have a good curative effect on the common cold, jaundice, gastric pain, diarrhea and ulcers ${ }^{8}$. A decoction of leaves, young shoots or the bark of A. trifoliatus has been used to treat tuberculosis and lung hemorrhages ${ }^{9}$. Some studies have indicated that extracts of $A$. trifoliatus possess several beneficial biological effects, including anti-inflammatory, immunostimulatory, and antioxidant properties, protein tyrosine phosphatase inhibitory activity and cytotoxic activities towards some types of cancer cells ${ }^{10-12}$.

${ }^{1}$ Department of Food Science and Technology, Jinan University, Guangzhou, 510632, China. ${ }^{2}$ Division of Research and Development, Kingmed Diagnostics, Guangzhou, 510330, China. ${ }^{3}$ These authors contributed equally: Qun Peng and Jianyuan Chen. *email: dhyjnu@sina.com; chao_wang@jnu.edu.cn 


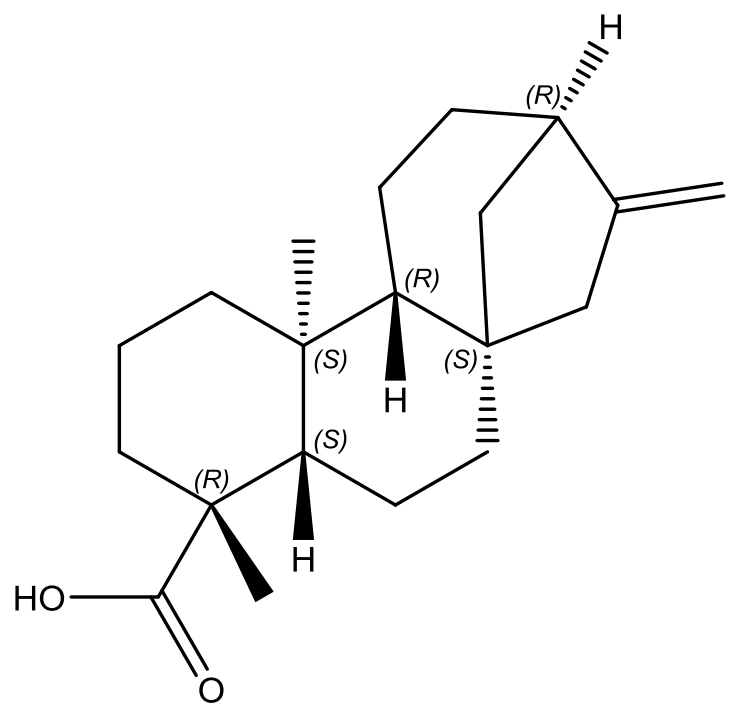

Figure 1. Chemical structure of KA.

Moreover, the young leaves and shoots of $A$. trifoliatus are popularly consumed as vegetables in traditional southern Chinese cuisine that can be stir-fried or cooked in a soup ${ }^{13}$. In addition, the local people of southern China have been using the leaves of this plant to make tea for daily consumption for at least 10 years for daily health management. This plant is also an ingredient in 'leicha', a traditional Chinese herbal tea that is believed to have a powerful tonic effect ${ }^{14}$. A. trifoliatus is usually planted from March to October and is a rigid shrub $1 \pm 0.4 \mathrm{~m}$ high. It is spiny with alternate palmate leaves and toothed margins, and it has a typical crisp flavor with some bitterness ${ }^{15}$.

The leaves of this plant contain a diverse range of components, including diterpenoids, lignans, triterpenoids, polyacetylenes, phenylpropanoids, and flavonoids ${ }^{16,17}$. Of these components, KA has been reported as an important ingredient in A. trifoliatus for its potent biological activities ${ }^{11,18-20}$. KA is a kaurane-type diterpenoid and is regarded as a key intermediate in the biogenesis of gibberellins and other phytohormones that regulate the growth and development of higher plants and some fungal metabolites ${ }^{21,22}$. The structure of KA is shown in Fig. 1 .

Therefore, for the effective utilization and quality control of natural resources, analysis of KA in A. trifoliatus could be very important. Several reports have determined KA contents by HPLC or GC. Early studies found that KA could not be completely separated from other components in plant extracts by HPLC because of its similar molecular structure and physical and chemical properties ${ }^{4,23,24}$.

UHPLC-MS/MS could be a useful tool to identify and quantify KA. In this study, a UHPLC-MS/MS method was developed for the quantitative determination of KA in the leaves of A. trifoliatus to use the leaves as an effective medical resource. Although the determination of KA by LC-MS/MS had been developed in early reports, this is the first fully validated method in terms of extraction, UHPLC-MS/MS conditions, linearity, precision, accuracy, limit of detection (LOD) and limit of quantitation (LOQ).

\section{Results and Discussion}

Effect of sample preparation on KA recovery. To investigate the influence on ultrasound time on the recovery of KA, $0.1 \mathrm{~g}$ of $A$. trifoliatus leaves were extracted in $100 \mathrm{~mL}$ of $100 \%$ methanol by ultrasonic extraction for 15,30 or $60 \mathrm{~min}$, and the determined concentrations were $4.1 \pm 0.3,4.2 \pm 0.3$ and $4.2 \pm 0.0 \mu \mathrm{g} / \mathrm{mg}$ solid, respectively. From these results, increasing the ultrasound treatment time slightly increased the extracted components but there was no significant difference after applying the ANOVA post-hoc test. Additionally, the R.S.D. of these three treatment times was $2.8-3.4 \%$. Therefore, a 15 min ultrasound time was chosen for further analysis.

Different sample extraction amounts were also optimized, including $0.1,0.2,0.3$ and $0.5 \mathrm{~g}$, and the corresponding results were $4.0 \pm 0.8,4.1 \pm 0.0,4.2 \pm 0.1$ and $4.1 \pm 0.1 \mu \mathrm{g} / \mathrm{mg}$ solid, respectively. The content of KA by weighting different sample extraction amounts were shown in Supplement 1. The R.S.D. of the four different sample extraction amounts was $2.3 \%$. By the ANOVA post-hoc test, there was no significant difference between the four exaction amounts. Therefore, to decrease the matrix effect, $0.1 \mathrm{~g}$ of sample was chosen in this study.

Optimization of UHPLC conditions on KA recovery. In this study, UHPLC conditions were mainly optimized in terms of the type of column, column temperature, flow rate and solvent effect. Two different UHPLC columns were tested with regards to method reproducibility, including an Accucore $\mathrm{C}_{18} \mathrm{UHPLC}_{\mathrm{H}}$ column $(100 \times 2.1 \mathrm{~mm}, 2.6 \mu \mathrm{m}$, Thermo Scientific, USA $)$ and a Zorbax Eclipse Plus $\mathrm{C}_{18}$ column $(2.1 \times 50 \mathrm{~mm}, 1.8 \mu \mathrm{m}$, Agilent Technologies, USA). The retention times of KA on the Accucore and Zorbax columns were 5.467 and $4.166 \mathrm{~min}$, respectively. Standard curves were constructed by using these two columns. The obtained standard curves were $\mathrm{Y}=136.7 \mathrm{X}+8.1\left(\mathrm{R}^{2}=0.9995\right.$, weight $\left.1 / \mathrm{x}\right)$ and $\mathrm{Y}=226.7 \mathrm{X}+86.8\left(\mathrm{R}^{2}=0.9739\right.$, weight $\left.1 / \mathrm{x}\right)$, where $\mathrm{X}$ : concentration $(\mathrm{ng} / \mathrm{mL})$ and $\mathrm{Y}$ : peak area. Thus, a much better linear relationship was obtained by using the Accucore column. This is probably because this column is longer than the Zorbax column, which gave good 
A.

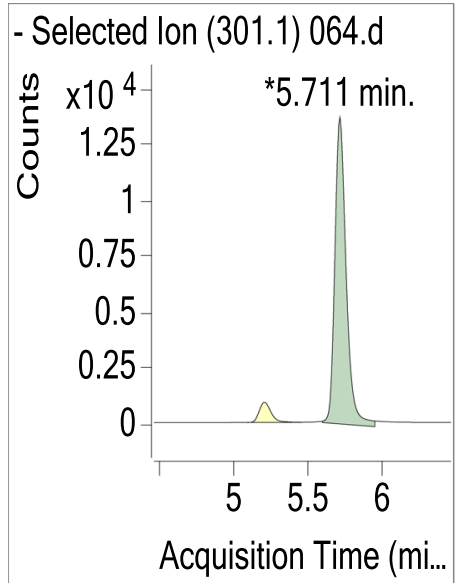

B.

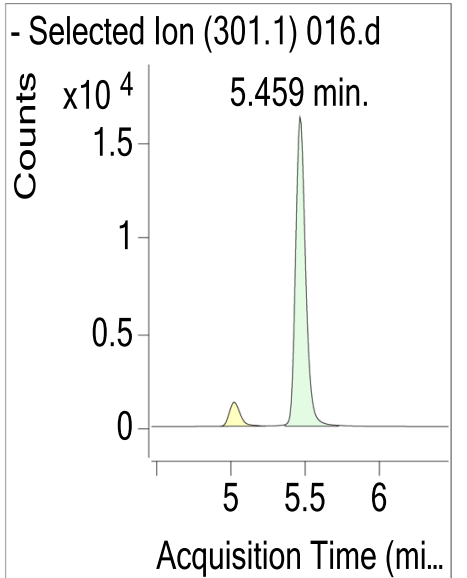

C.

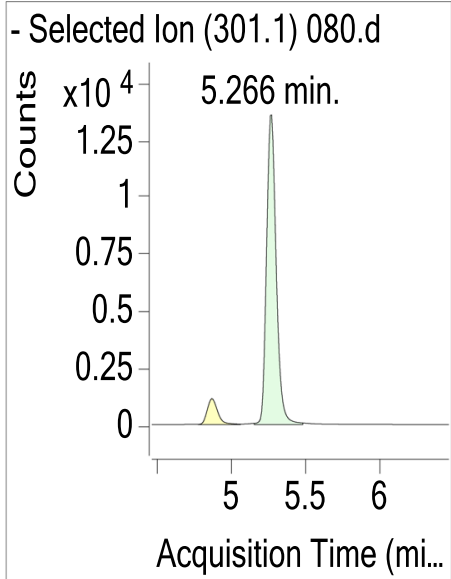

Figure 2. Effect of flow rate on the UHPLC-MS/MS chromatogram of the KA extract from A. trifoliatus leaves. (A) $0.3 \mathrm{~mL} / \mathrm{min},\left(\right.$ B) $0.4 \mathrm{~mL} / \mathrm{min},(\mathbf{C}) 0.45 \mathrm{~mL} / \mathrm{min}$. KA was separated on an Accucore $\mathrm{C}_{18} \mathrm{LC}$ column and quantified with MRM transition at the quantitative fragment ion/product ion $(\mathrm{m} / \mathrm{z}: 301.3 / 301.3)$ in ESI negative mode.

A.

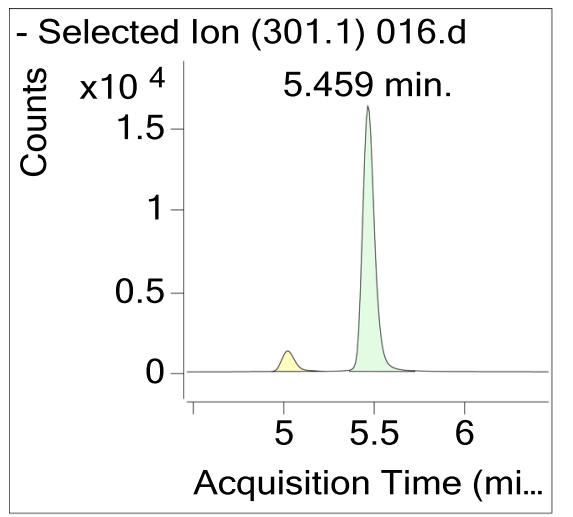

B.

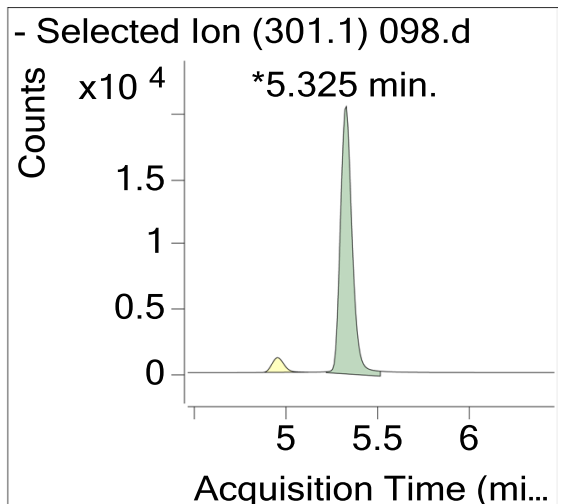

Figure 3. Effect of column temperature on the UHPLC-MS/MS chromatogram of the KA extract from $A$. trifoliatus leaves. (A) $35^{\circ} \mathrm{C}$, (B) $40^{\circ} \mathrm{C}$. KA was separated on an Accucore $\mathrm{C}_{18} \mathrm{LC}$ column at a flow rate of $0.4 \mathrm{~mL} /$ min and quantified with MRM transition at the quantitative fragment ion/product ion $(\mathrm{m} / z: 301.3 / 301.3)$ in ESI negative mode.

separation from the interfering substances to decrease the matrix effect and achieve higher reproducibility. Therefore, the Accucore column was chosen for further study.

Three flow rates were tested for their method reproducibility, including $0.35,0.4$ and $0.45 \mathrm{~mL} / \mathrm{min}$. Three UHPLC-MS/MS chromatograms were obtained as shown in Fig. 2. From the figure, the KA peak shape was sharp and symmetrical with no tailing and very high resolution. The obtained analyte concentrations using the three flow rates were $4.0 \pm 0.1,4.0 \pm 0.1$, and $4.1 \pm 0.2 \mu \mathrm{g} / \mathrm{mg}$ solid, and there were no significant differences in the test results between these three columns by the ANOVA post-hoc test. Therefore, from our results, 0.4 or $0.45 \mathrm{~mL} / \mathrm{min}$ could be chosen as the flow rate, and we used $0.45 \mathrm{~mL} / \mathrm{min}$ as the flow rate. The base peak width was reduced to $0.12 \mathrm{~min}$ when using $0.45 \mathrm{~mL} / \mathrm{min}$ as the flow rate. By reducing the peak width, this could be utilized to enable faster analysis times while maintaining resolution.

Two different column temperatures were tested on method reproducibility, including 35 and $40^{\circ} \mathrm{C}$, and their corresponding UHPLC-MS/MS chromatograms are shown in Fig. 3. From the figure, both KA peak shapes are sharp and symmetrical with no tailing, and very high resolution, and there were no other interferences. The retention times from the two column temperatures were 5.46 and $5.32 \mathrm{~min}$ for $35^{\circ} \mathrm{C}$ and $40^{\circ} \mathrm{C}$, respectively. The obtained analyte concentrations at the two temperatures were $4.0 \pm 0.1$ and $4.5 \pm 0.5 \mu \mathrm{g} / \mathrm{mg}$ solid, and there was no significant difference between these two analyses. Therefore, in this study, we chose $35^{\circ} \mathrm{C}$ as our column temperature.

As indicated previously, electrospray solvent has a significant impact on the chromatogram and mass spectra of tandem mass spectrometry ${ }^{25}$. In this study, KA was extracted from A. trifoliatus leaves with $100 \%$ methanol. 
A.

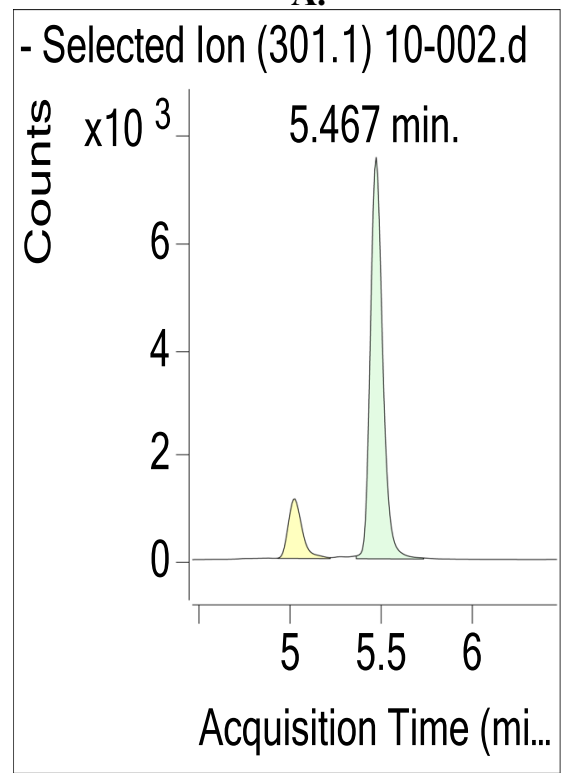

B.

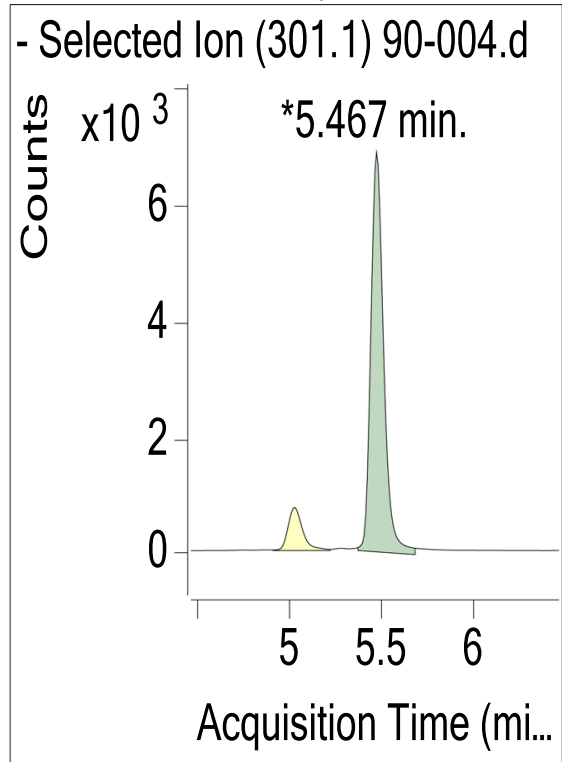

Figure 4. Effect of electrospray solvent on the UHPLC-MS/MS chromatogram of the KA extract from Acanthopanax trifoliatus leaves. (A) 2\% FA in methanol; (B) 2\% FA in 50\% methanol. KA was separated on an Accucore $\mathrm{C}_{18} \mathrm{LC}$ column at a flow rate of $0.4 \mathrm{~mL} / \mathrm{min}$ and quantified with $\mathrm{MRM}$ transition at the quantitative fragment ion/product ion $(m / z: 301.3 / 301.3)$ in ESI negative mode. UHPLC column temperature: $35^{\circ} \mathrm{C}$.

However, to improve the precision of the standard curve, $50 \%$ methanol was applied as a dilution solvent to prepare the standard solutions, and their corresponding UHPLC-MS/MS chromatograms are shown in Fig. 4. From the figure, the retention time from the two solvents was the same. The obtained concentration of analyte from the two solvents was also not significantly different.

In this study, we applied MRM as our MS/MS function. Very uniquely, we selected ion $\mathrm{m} / \mathrm{z} 301.3$ on Q1 by applying the appropriate Q2 (collision energy, $30 \mathrm{eV}$ ), and Q3 was obtained, but the product ion remained 301.3. This phenomenon indicated that KA lacked fragmentation under collision. Therefore, we chose the MRM transition as $301.3>301.3$. These unique characteristics were also observed by another study ${ }^{26}$. Different collision energies were tested on $\mathrm{KA}$ from $0 \mathrm{eV}$ to $40 \mathrm{eV}$ and there was no unique fragments observed. Also the product ion with $\mathrm{m} / \mathrm{z} 301.2$ was the only ion detected when charging the collision energy from 0 to $40 \mathrm{eV}$ on the Q2 which were shown in Supplement 2.

Method validation. KA was detected in negative ion mode and was well-separated on the reversed phase column used. The KA molecule exhibited an adequately separate chromatographic peak that was easily distinguished from the baseline. Quantitation was performed based on retention time, which was compared with the authentic standard MRM transition.

Method selectivity was assessed by analyzing a blank, a positive sample and a KA standard solution. Selectivity met the criterion because there were no interfering peaks observed at the same retention times as the target analytes.

The calibration curve showed an acceptable linearity range and $\mathrm{R}^{2}$. For the equation $\mathrm{Y}=136.7 \mathrm{X}+8.1, \mathrm{X}$ is in the range of $8 \mathrm{ng} / \mathrm{mL} \sim 160 \mathrm{ng} / \mathrm{mL}$, the weight is $1 / x$, and $\mathrm{R}^{2}=0.9995$. No unacceptable carryover was observed. Homoscedasticity should be tested in any linear regression analysis, especially for an analytical method in which the concentration range is broad ${ }^{27}$. By constructing the residual vs. concentration plot and applying the $F$-test, our calibration data did not meet the homoscedasticity requirement because the residuals are not randomly distributed. Thus, a weighted calibration curve should be constructed. Two different weighting factors $\left(1 / \mathrm{x}\right.$ and $\left.1 / \mathrm{x}^{2}\right)$ are compared, and the data are shown in Table $1.1 / \mathrm{x}$ showed a smaller $\% \mathrm{RE}$ than $1 / \mathrm{x}^{2}$, and thus, the $1 / \mathrm{x}$ weighting factor was chosen.

The LOQ was determined as $4 \mathrm{pg}$ which was obtained by injection $0.5 \mu \mathrm{L}$ of $8 \mathrm{ng} / \mathrm{mL} \mathrm{KA}$ solution. At this concentration, signal to baseline noise $(S / N)$ ratio was over 10 and bias $(\%)$ in accuracy and precision were below $20 \%$. Lower concentration $(6 \mathrm{ng} / \mathrm{mL})$ was tested and found the precision was $35 \%$ and $S / N<10$. Therefore LOQ was considered as the lowest calibration standard and be quantified reliably. The LOD was determined by $S / N$ ratio of 3 and found LOD was $1.3 \mathrm{pg}$. These results were lower than those from an early report ${ }^{26}$.

Intra-day, inter-day and inter-person method repeatability were evaluated for 9 replicates. The obtained KA in the extract for the intra-day analysis by the same operator was $4.1 \pm 0.1 \mu \mathrm{g} / \mathrm{mg}$ solid, and the R.S.D. was $2.8 \%$. The obtained KA in the extract for the inter-day analysis by the same operator was $4.0 \pm 0.1 \mu \mathrm{g} / \mathrm{mg}$ solid, and the R.S.D. was $3.2 \%$. The values of KA concentration from operator 1 and operator 2 were $4.0 \pm 0.1 \mu \mathrm{g} / \mathrm{mg}$ solid and $4.2 \pm 0.1 \mu \mathrm{g} / \mathrm{mg}$ solid, respectively. The R.S.D. of inter-person was 3.6\%. The KA in the extract obtained by 


\begin{tabular}{|l|l|l|l|}
\hline \multirow{2}{*}{ Concentration $(\mathbf{n g} / \mathbf{m L})$} & \multirow{2}{*}{ No weighting } & $\mathbf{1}$ weighting \\
\cline { 3 - 4 } & 259 & 110 & $\mathbf{1} / \mathbf{x}^{\mathbf{2}}$ \\
\hline 8 & 110 & 86 & 197 \\
\hline 16 & 74 & 40 & 51 \\
\hline 40 & 10 & 13 & 15 \\
\hline 80 & 15 & 14 & 17 \\
\hline 160 & 468 & 263 & 375 \\
\hline Sum \% RE & & \multicolumn{2}{|l}{} \\
\hline
\end{tabular}

Table 1. Sum of the relative error (RE) of 3 replicates for KA at different standard concentrations.

\begin{tabular}{|l|l|l|}
\hline QC concentration $(\boldsymbol{\mu} \mathbf{g} / \mathbf{m L})$ & Matrix effect $(\%)$ & Intra-day accuracy $(\%)$ \\
\hline 1.81 & $95.2(1.2)$ & $99.2(8.0)$ \\
\hline 3.62 & $93.8(2.4)$ & $99.7(2.7)$ \\
\hline 6.03 & $95.4(3.2)$ & $97.0(5.9)$ \\
\hline
\end{tabular}

Table 2. Accuracy and matrix effect for KA at different standard concentrations. Standard deviation was given in the parentheses.

different instruments of the same model (Agilent 6460 ) on the same day was $4.1 \pm 0.1 \mu \mathrm{g} / \mathrm{mg}$ solid, and the R.S.D. was $2.9 \%$. Therefore, all precision values were below $15 \%$, which met the EMA guideline requirements ${ }^{28}$.

Accuracy was expressed as the recovery, determined by analyzing 3 replicates of three quality controls (QCs) spiked in the sample for extract. The three quality controls (QCs) were prepared from the KA standard stock solutions corresponding to 3 points, a low QC, medium QC and high QC. The final concentrations of three QCs were $1.81,3.62$ and $6.03 \mu \mathrm{g} / \mathrm{mg}$ solid. The recoveries (\%) were 99.2 $\pm 8.0,99.7 \pm 2.7$ and $97 \pm 5.9$. The detailed results about accuracy test were shown in Supplement 3. This shows that the method met the requirements of the EMA guidelines and that the recovery was in the range of $75 \sim 110 \%{ }^{28}$. A minor matrix effect of $94 \%$ was observed with the extract as the matrix. MAX SPE purification significantly decreased the matrix effect. The accuracy and matrix effect results were shown in Table 2.

\section{Conclusions}

In this study, a UHPLC-MS/MS method was developed as a new assay procedure and was evaluated and applied for specific quantitation of KA content in A. trifoliatus leaves after purification by a SPE cartridge. After the implementation of an ultrasound-assisted extraction, this UHPLC-MS/MS method was validated in-house with the plant extract demonstrating good recovery rates above $97 \%$ and good R.S.D. This method showed very good selectivity and sensitivity with a LOD of $1.3 \mathrm{pg}$ and a LOQ of $4 \mathrm{pg}$. The method has been applied successfully in the determination of KA content in A. trifoliatus leaves, which can reach $0.4 \%$ ( $4 \mu \mathrm{g} / \mathrm{mg}$ solid).

\section{Methods}

Chemicals. LC-MS grade water (LC-MS, LiChrosolv), acetonitrile (LC-MS, LiChrosolv), methanol (LC-MS, LiChrosolv) and formic acid (reagent grade, 95\%) were obtained from Sigma-Aldrich (Shanghai, China). The KA standard with a purity over $98 \%$ was obtained from the National Drug Reference Standards Center (Beijing, China). A. trifoliatus leaves were picked in April 2018 from a farm in Enping, Guangdong Province and shipped to Jinan University on the same day under refrigerated conditions. Upon arriving at the lab, the fresh leaves of A. trifoliatus were washed, dried in an air flow, ground in liquid nitrogen, lyophilized for 2 days and then powdered with an electronic mill (20-mesh sieve). The powder was kept in centrifuge tubes sealed with parafilm and reserved in dry and cool conditions until analysis.

Extraction KA from A. trifoliatus. The extraction procedure of KA from A. trifoliatus Merr. was optimized for plant leaf powder in terms of sample mass and ultrasound-assisted extraction time. A set of three replicates of plant leaf powder were weighed into a volumetric flask with a cap, and $100 \mathrm{~mL}$ methanol was added as the extraction solvent. The mixture was first vortexed and then sonicated for a certain amount of time, including 15, 30 and $60 \mathrm{~min}$. An ultrasonic water bath (Powder sonic 420, with a $40 \mathrm{kHz}$ frequency, maximum power of $700 \mathrm{~W}$, and internal dimensions (id) of $500 \times 300 \times 150 \mathrm{~mm}$ ) was utilized for this purpose. After sonication, the mixture was cooled to room temperature and then passed through vacuum filter paper (Whatman qualitative filter paper, Grade 1, Sigma-Aldrich), and the supernatant was recovered. Different amounts of powder were tested for extraction, including $0.1,0.2,0.3$ and $0.5 \mathrm{~g}$. Ultrasound-assisted extraction times included 15,30 and $60 \mathrm{~min}$. The $5 \mathrm{~mL}$ of extract was evaporated completely using a vacuum evaporator centrifuge (Labconco, Fisher Scientific, USA), dissolved in $10 \mathrm{~mL}$ of water and cleaned up by solid phase extraction (SPE).

SPE clean up. The KA extract was further cleaned up by an Oasis MAX (mixed-mode anion exchange, $60 \mathrm{mg}, 3 \mathrm{cc}$, Waters) cartridge column for acidic compounds. The Oasis MAX cartridge was first activated and conditioned with $10 \mathrm{~mL}$ of methanol and $10 \mathrm{~mL}$ of Mill-Q water. Then, $0.25 \mathrm{~mL}$ of the extract was applied to the cartridge. Next, the column was washed with $10 \mathrm{~mL}$ of $5 \% \mathrm{NH}_{4} \mathrm{OH}$ in water and dried for 5 min under a stream of 
air. The analytes were then eluted with $10 \mathrm{~mL}$ of $2 \% \mathrm{FA}$ in methanol and further filtered through a $0.22 \mu \mathrm{m}$ nylon filter before injection into the UHPLC-MS/MS. The extract was diluted 80-fold to obtain an extract concentration in the range of the linear standard curve.

UHPLC-MS/MS conditions. Chromatographic separation of KA from the other matrix components was carried out by reversed phase chromatography on an Agilent 1290 Infinity UHPLC equipped with a binary pump, autosampler, column oven and degasser (Agilent Corp., Santa Clara, CA). The mobile phase was constituted as follows: $0.1 \%$ FA in water (solution A) and acetonitrile (solution B). For the determination of KA, different chromatographic columns were tested in this study, including an Accucore $\mathrm{C}_{18} \mathrm{LC}$ column $(100 \times 2.1 \mathrm{~mm}, 2.6$ $\mu \mathrm{m}$, Thermo Scientific, USA) and a Zorbax Eclipse Plus $\mathrm{C}_{18}$ column $(2.1 \times 50 \mathrm{~mm}, 1.8 \mu \mathrm{m}$, Agilent Technologies, USA). Different flow rates for KA recovery and method precision were tested, including $0.35,0.4$ and $0.45 \mathrm{~mL} /$ min. KA chromatographic separation was performed using the following gradient elution: $0-1 \mathrm{~min}, 90 \% \mathrm{~A}$; $1-3 \mathrm{~min}, 90 \% \mathrm{~A}$ to $60 \% \mathrm{~A} ; 3-4 \mathrm{~min}, 60 \% \mathrm{~A}-10 \% \mathrm{~A} ; 4-6.5 \mathrm{~min}, 10 \%$, then return to the initial conditions and equilibrate the column for $1 \mathrm{~min}$. The injection volume was $0.5 \mu \mathrm{L}$, the autosampler was kept at $20^{\circ} \mathrm{C}$ and column temperature was investigated at $35^{\circ} \mathrm{C}$ and $40^{\circ} \mathrm{C}$.

The UHPLC eluate was interfaced with an Agilent 6460 triple quadrupole mass spectrometer (Agilent Corp.) operating in negative ion electrospray mode. An Agilent Mass Hunter workstation was used for control of the equipment, data acquisition and analysis. For optimization of the MS/MS parameters, a KA standard solution prepared in methanol was infused into the mobile phase $(0.5 \mathrm{mg} / \mathrm{mL})$ at a flow rate of $20 \mu \mathrm{L} / \mathrm{min}$ using a syringe pump (Harvard Apparatus, Holliston, MA) to tune the instrument. Nitrogen was used both as the drying gas at a flow rate of $11 \mathrm{~L} / \mathrm{min}$ and as the nebulizing gas at a pressure of $45 \mathrm{psi}$. The drying gas temperature was $300^{\circ} \mathrm{C}$, and a potential of $4 \mathrm{kV}$ was applied across the capillary. Finally, the instrument was operated with a fragmentor voltage of $140 \mathrm{~V}$. Acquisition was performed by MRM, wherein the respective pseudomolecular anion of KA was fragmented by collision-induced dissociation into a fragment for detection. The MRM transition and the collision voltage were optimized, and the value of the collision energy for the qualitative and quantitative ion (301.1) was $30 \mathrm{eV}$. The dwell time was $100 \mathrm{~ms}$.

Method validation. The developed method was validated based on the EMA guidelines, including selectivity, carryover, limit of detection and limit of quantification, linearity, repeatability, precision, accuracy and matrix effect ${ }^{28}$.

Calibration curves and linearity. (1) KA stock solutions were prepared by fully dissolving $1 \mathrm{mg}$ of KA into a $10 \mathrm{~mL}$ brown volumetric flask in methanol. The concentration of the stock solution was $0.1 \mathrm{mg} / \mathrm{mL}$. The stock solution was completely mixed and stored at $-80^{\circ} \mathrm{C}$. (2) KA intermediate standard solutions were prepared by pipetting $1 \mathrm{~mL}$ of the stock solution into a $25 \mathrm{~mL}$ brown volumetric flask and bringing up to volume with $50 \%$ methanol. The final concentration of the intermediate standard solution was $4 \mu \mathrm{g} / \mathrm{mL}$. The intermediate standard solutions were also stored at $-80^{\circ} \mathrm{C}$. (3) The working solutions of KA were prepared by pipetting $20 \mu \mathrm{L}, 40 \mu \mathrm{L}$, $100 \mu \mathrm{L}, 200 \mu \mathrm{L}$ and $400 \mu \mathrm{L}$ of the intermediate solutions into a $10 \mathrm{~mL}$ brown volumetric flask and bringing up to volume with $50 \%$ methanol. The final concentrations of the working solutions were $8,16,40,80$ and $160 \mathrm{ng} / \mathrm{mL}$. The ranges of the calibration curves were determined from the preliminary analysis of KA in A. trifoliatus leaves. A $1 / x$ weighted linear regression was applied so that the back-calculated concentration should be within $15 \%$ of the theoretical concentration.

Solvent effects in tandem mass spectrometry. An early study discovered that chromatograms and mass spectra may not only be dependent on careful control of instrumental parameters such as tuning conditions, but also on the electrospray solvent ${ }^{25}$. In this study, KA was extracted from A. trifoliatus leaves with methanol as the extraction solvent. However, to improve the precision of the standard curve, 50\% methanol was applied as the dilution solvent to prepare the standard solutions. Therefore, the chromatograms of KA in methanol and 50\% methanol were compared in terms of peak shape, peak area and retention time.

The LOQ was considered as the lowest concentration of analytes that can be quantified reliably so that the bias (\%) in accuracy and precision were below 20\%. A signal-to-ratio of 3 and 10 is used for estimating LOD and LOQ, respectively. The LOQ and LOD were determined by diluting the KA standard solution until the $S / N$ ratio reached to 10 and 3 , respectively.

Selectivity and carryover. The selectivity refers to the method that can differentiate the target analyte from other matrix components. This was assessed by analyzing negative samples, positive samples and KA standard solutions. The presence of possible endogenous or exogenous interferences was verified by monitoring the MRM chromatogram. The MRM chromatogram was specific for KA at its expected retention time. The acceptance criterion for the selectivity was that the peak area of interference should be below $20 \%$ of the peak area of the LOQ of KA.

Carryover was evaluated by injecting blank samples after the run of the highest calibration concentration. The acceptance criteria for carryover were the same as for the evaluation of selectivity.

Robustness. To assess the robustness of the method, 9 replicates of the sample extraction were prepared by the same operator under the same instrument on the same day.

Precision. The precision was evaluated by the precision of 2 different operators on 3 nonconsecutive days, and by two instruments of the same model with 9 replicates of sample extraction. 
Accuracy. The accuracy was expressed as the recoveries, determined by analyzing 3 replicates of three quality controls (QCs) spiked in the sample for extract. Three quality controls (QCs) were prepared from the KA standard stock solutions with 3 points, low QC, medium QC and high QC. The final three QCs were 1.81, 3.62 and $6.03 \mu \mathrm{g} / \mathrm{mg}$ solid, which corresponded to $0.5-, 1$ - and 1.5-fold the KA concentration in the plant extract. $0.5 \mathrm{~mL}$ individual QC stock solution $(362,724$ and $1206 \mu \mathrm{g} / \mathrm{mL})$ was spiked in the same amounts of samples and gone through ultrasound-assisted extraction, concentration and SPE clean-up. The final extracts were determined in the same day. The accuracy (recovery) was calculated with the following equations:

$$
\operatorname{Accuracy}(\%)=\frac{(A-B)_{*} 100}{C}
$$

A: KA concentration in sample extracts spiked with individual QC standard solution; B: KA concentration in sample extracts; C: nominal value of KA in QC standard solution. The KA concentration obtained from A and B were calculated against the calibration curve.

Matrix effect. The matrix effect was also determined for the method. The sample matrix, such as coeluting compounds, can contribute to alterations in the analyte ionization and overall response. Therefore, complete separation between the analyte and the coeluting matrix could help to decrease or increase the ionization of the target analyte. The matrix effect was determined with the following method. The volume of $10 \mu \mathrm{L}$ QCs $(1.81,3.62$ and $6.03 \mu \mathrm{g} / \mathrm{mL}$ ) were spiked into $1 \mathrm{~mL}$ post sample extract. The peak area obtained in the matrix was compared with the corresponding peak area in the extraction solution with the same concentration. Each sample was analyzed three times. The matrix effect was calculated as follows:

$$
\text { Matrix effect }(\%)=(A-B) \times 100 / C
$$

A: mean peak area in the matrix after spiking the QCs; B: endogenous KA peak area in the matrix before spiking the QCs; and C: mean peak area of three QCs in the extraction solution.

Received: 12 November 2018; Accepted: 11 February 2020;

Published online: 25 February 2020

\section{References}

1. Bucci, L. R. Selected herbals and human exercise performance. Am J. Clin. Nutr. 72, 624s-636s (2000).

2. Kiem, P. V., Cai, X. F., Van Minh, C., Lee, J. J. \& Kim, Y. H. Kaurane-type diterpene glycoside from the stem bark of Acanthopanax trifoliatus. Planta Med. 70, 282-284 (2004).

3. Chien, T. M. et al. Acanthopanax trifoliatus inhibits lipopolysaccharide-induced inflammatory response in vitro and in vivo. Kaohsiung J. Med Sci. 31, 499-509 (2015).

4. Hu, H. B., Zheng, X. D. \& Hu, H. S. Chemical composition, antimicrobial, antioxidant and cytotoxic activity of the essential oil from the leaves of Acanthopanax leucorrhizus (Oilv.) Harms. Environ. Toxicol. Phar. 34, 618-623 (2012).

5. Ministry of ScienceTechnology and Environment Red Data Book of Vietnam, Vol. 2- Plants, Science and Techniques Publishing House, Hanoi, (1996).

6. Sithisarn, P., Rojsanga, P., Jarikasem, S., Tanaka, K. \& Matsumoto, K. Ameliorative Effects of Acanthopanax trifoliatus on Cognitive and Emotional Deficits in Olfactory Bulbectomized Mice: An Animal Model of Depression and Cognitive Deficits. Evid-Based Compl. Alt. Med. 1-9 (2013).

7. Zhang, X. D., Liu, X. Q., Kim, Y. H. \& Whang, W. K. Chemical constituents and their acetyl cholinesterase inhibitory and antioxidant activities from leaves of Acanthopanax henryi: potential complementary source against Alzheimer's disease. Arch. Pharm. Res. 37, 606-616 (2014)

8. Wang, H. Q. et al. Antioxidant and anti-inflammatory properties of Chines ilicifolius vegetable (Acanthopanax trifoliatus (L) Merr.) and its reference compounds. Food Sci. Biotechnol. 24, 1131-1138 (2015).

9. Sithisarn, P., Muensaen, S., Jarikasem, S. \& Supatanakul, W. Antioxidant Activity and Phenolic Contents of Extracts from the Leaves of Acanthopanax trifoliatus Harvested in Different Seasons. Acta Hortic. 1023, 167-171 (2014).

10. Wang, H. Q. et al. Anticancer Activity of Acanthopanax trifoliatus (L) Merr Extracts is Associated with Inhibition of NF-kappa B Activity and Decreased Erk1/2 and Akt Phosphorylation. Asian Pac. J. Cancer P. 15, 9341-9346 (2014).

11. Li, D. L. et al. Terpenoid composition and the anticancer activity of Acanthopanax trifoliatus. Arch. Pharm. Res. 39, 51-58 (2016).

12. Sithisarn, P., Jarikasem, S. \& Thisayakorn, K. Anti-inflammatory and antioxidative effects of leaf extract from Acanthopanax trifoliatus. Planta Med. 75, 891-891 (2009).

13. Sithisarn, P., Muensaen, S. \& Jarikasem, S. Determination of Caffeoyl Quinic Acids and Flavonoids in Acanthopanax trifoliatus Leaves by HPLC. Nat. Prod. Commun. 6, 1289-1291 (2011).

14. Roslida, A. H., Siti, Nurusaadah., H. \& Fezah., O. Gastroprotective effect of Acanthopanax trifoliatus on experimentally induced acute ulcer in rats. Pharmacologyonline. 2, 828-841 (2010).

15. Agence de Cooperation Culturelle et Technique. Medecine Traditionnelle et Pharmacopee: Les Plantes Medicinales au Vietnam, Paris Tome 1, 23 (1996).

16. Sithisarn, P., Jarikasem, S. \& Muensaen, S. Comparative HPLC analysis of phenolic compounds in the roots of Acanthopanax trifoliatus and Acanthopanax senticosus. Ac. 1125, 19-24 (2016).

17. Van Kiem, P., Cai, X. F., Van Minh, C., Lee, J. J. \& Kim, Y. H. Lupane-triterpene carboxylic acids from the leaves of Acanthopanax trifoliatus. Chem. Pharm. Bull. 51, 1432-1435 (2003).

18. Wilkens, M., Alarcon, C., Urzua, A. \& Mendoza, L. Characterization of the bactericidal activity of the natural diterpene kaurenoic acid. Planta Med. 68, 452-454 (2002).

19. Cunha, K. M. D. et al. Smooth muscle relaxant effect of kaurenoic acid, a diterpene from Copaifera langsdorffii on rat uterus in vitro. Phytother Res. 17, 320-324 (2003).

20. Cavalcanti, B. C. et al. Genotoxicity evaluation of kaurenoic acid, a bioactive diterpenoid present in Copaiba oil. Food Chem. Toxicol. 44, 388-392 (2006).

21. Sun, T. P. \& Kamiya, Y. The Arabidopsis GA1 locus encodes the cyclase ent-kaurene synthetase A of gibberellin biosynthesis. The Plant cell. 6, 1509-18 (1994).

22. Yamaguchi, S., Sun, T., Kawaide, H. \& Kamiya, Y. The GA2 locus of Arabidopsis thaliana encodes ent-kaurene synthase of gibberellin biosynthesis. Plant physiology. 116, 1271-8 (1998). 
23. Phuong, N. T. et al. Capillary electrophoretic method for the determination of diterpenoid isomers in Acanthopanax species. J. Pharm. Biomed. Anal. 40, 56-61 (2006).

24. Zhang, X., Zhou, X., Liu, X., Li, X. \& Whang, W. Development and Application of an HPLC-UV Procedure to Determine Multiple Flavonoids and Phenolics in Acanthopanax Leaf Extracts. J. Chromatogr. Sci. 54, 574-82 (2016).

25. Desaire, H., Leavell, M. D. \& Leary, J. A. Solvent effects in tandem mass spectrometry: mechanistic studies indicating how a change in solvent conditions and pH can dramatically alter CID spectra. J. Org. Chem. 67, 3693-9 (2002).

26. Miyazaki, S. et al. Analysis of ent-kaurenoic acid by ultra-performance liquid chromatography-tandem mass spectrometry. Biochem. Biophys Rep. 2, 103-107 (2015).

27. Almeida, A. M., Castel-Branco, M. M. \& Falcao, A. C. Linear regression for calibration lines revisited: Weighting schemes for bioanalytical methods. J. Chromatogr. B. 774, 215-222 (2002).

28. European Medicine Agency. Guideline on bioanalytical method validation (2011).

\section{Acknowledgements}

This research received financial support from Guangdong Natural Science Foundation (No. 2018A030313091).

\section{Author contributions}

The study was conceived by C.W. and HY. D. Q.P. performed the sample preparation and data analysis. JY. C. performed the UHPLC-MS/MS method development and analysis. Q.P. and JY. C. wrote the manuscript with input from C.W. and HY. D. All the authors discussed the results and commented on the manuscript.

\section{Competing interests}

The authors declare no competing interests.

\section{Additional information}

Supplementary information is available for this paper at https://doi.org/10.1038/s41598-020-60426-3.

Correspondence and requests for materials should be addressed to H.D. or C.W.

Reprints and permissions information is available at www.nature.com/reprints.

Publisher's note Springer Nature remains neutral with regard to jurisdictional claims in published maps and institutional affiliations.

(c) (1) Open Access This article is licensed under a Creative Commons Attribution 4.0 International License, which permits use, sharing, adaptation, distribution and reproduction in any medium or format, as long as you give appropriate credit to the original author(s) and the source, provide a link to the Creative Commons license, and indicate if changes were made. The images or other third party material in this article are included in the article's Creative Commons license, unless indicated otherwise in a credit line to the material. If material is not included in the article's Creative Commons license and your intended use is not permitted by statutory regulation or exceeds the permitted use, you will need to obtain permission directly from the copyright holder. To view a copy of this license, visit http://creativecommons.org/licenses/by/4.0/.

(C) The Author(s) 2020 